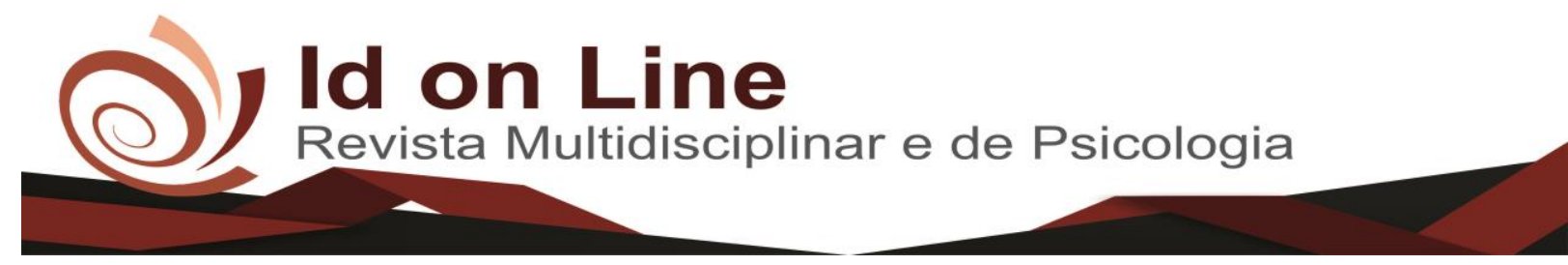

Artigo de Revisão

\title{
Satisfação dos usuários de serviços públicos odontológicos: Revisão da literatura
}

\author{
Luciana Cristina Salvador Azevedo ${ }^{1}$; Marcelo Pereira da Rocha ${ }^{2}$
}

\begin{abstract}
Resumo: A satisfação dos usuários vem sendo utilizada como ferramenta na melhoria dos serviços de saúde bucal: possibilita a Identificação de limitações e melhor entendimento do perfil do paciente; contribuindo para aprimoramento das práticas profissionais e garantindo a participação social. Objetivo: Avaliar a satisfação dos usuários de serviços públicos odontológicos. Metodologia: Revisão da literatura. Os artigos foram selecionados utilizando as bases de dados LILACS e SCIELO através das palavras-chave: satisfação dos usuários; Odontologia; SUS. Foram incluídos artigos da língua inglesa e portuguesa de 1988 a 2018. Resultados: Num total de nove artigos selecionados, observou-se elevado nível de satisfação pela humanização do atendimento. Por outro lado, $70 \%$ dos pacientes classificaram o acesso ao serviço como difícil ou muito difícil. A estrutura física foi categorizada como satisfatória, bem organizada e higienizada. Conclusão: A satisfação dos usuários pode ser citada como excelente, porém novos estudos são necessários para aprofundamento no tema.
\end{abstract}

Palavras-chave: satisfação dos usuários; Odontologia; Sistema Único de Saúde (SUS)

\section{Satisfaction of users of dental public services: Literature review}

\begin{abstract}
The satisfaction of the users has been used as a tool in the improvement of oral health services: it allows the Identification of limitations and better understanding of the profile of the patient; contributing to the improvement of professional practices and ensuring social participation. Objective: To evaluate the satisfaction of users of public dental services. Methodology: Literature review. The articles were selected using the LILACS and SCIELO databases through the following keywords: user satisfaction; Dentistry; SUS. English and Portuguese articles were included from 1988 to 2018. Results: Nine selected articles showed a high level of satisfaction with the humanization of care. On the other hand, $70 \%$ of the patients classified the access to the service as difficult or very difficult. The physical structure was categorized as satisfactory, well organized and sanitized. Conclusion: User satisfaction can be classified as excellent, but further studies are needed to delve into the topic.
\end{abstract}

Keywords: user satisfaction; Dentistry; Unified Health System

\footnotetext{
${ }^{1}$ Graduanda em Odontologia pela Faculdade Independente do Nordeste, Vitória da Conquista, Bahia. Brasil. Contato: lucianaazevedo29@hotmail.com

2 Mestre em Saúde Coletiva, Faculdade Independente do Nordeste, Vitória da Conquista, Bahia, Brasil. cdbiomarcelo@yahoo.com.br
} 


\section{Introdução}

O interesse pela satisfação dos usuários no setor saúde teve início na década de 70 , baseado em aspectos técnicos e estruturais da qualidade da atenção. Há várias décadas esse tema vem sendo considerado um componente importante na qualidade do cuidado, bem como provedor de informações confiáveis e adequadas sobre a opinião do público em relação aos serviços oferecidos (CORBACHO, 2011).

Estudos sobre a satisfação do usuário são importantes, pois avaliam a qualidade dos serviços de saúde e fornecem subsídios para os administradores e cuidadores que ali trabalham, possibilitando a superação das limitações detectadas (D’ÁVILA et. al., 2010).

A opinião do usuário sobre os serviços prestados é a chave para o sucesso da adesão ao tratamento. O papel do usuário como protagonista do sistema de saúde tem impacto direto na melhoria do serviço. Assim, é fundamental conhecer como eles avaliam o atendimento prestado, para repensar as práticas profissionais ou intervir através da organização e aprimoramento dos serviços (MATOS e TOMITA, 2010).

Os níveis de saúde/doença da comunidade podem complementar esse tipo de avaliação. Este processo é descrito por Donabedian (1990) como mudanças no estado de saúde atual e futuro do paciente, podendo estas serem sociais, psicológicas, fisiológicas, físicas como também de atitude, satisfação e de comportamento (DONABEDIAN, WHEELER e WYSZEWIANSKI, 1982).

Conhecer a percepção dos usuários a respeito da qualidade do que lhe é ofertado é uma boa forma para os gestores conhecerem e desenvolverem ações que levem a melhorias contínuas, configurando-se como uma importante ferramenta na construção eficaz no direcionamento de planos e estratégias (MATOS e TOMITA, 2010; DONABEDIAN, 1990).

O vínculo verdadeiramente participativo e de responsabilidade com a população faz toda a diferença durante o processo (MOYSÉS, 2009), que não deve ser encarado como obrigação, mas como exercício de um direito, que já é garantido por uma das diretrizes do SUS: a participação social. 


\section{Metodologia}

A metodologia a ser utilizada para a elaboração desta pesquisa será a de revisão de literatura, buscando-se uma síntese do conhecimento acerca da satisfação dos usuários dos serviços do SUS.

Para tanto, serão utilizadas as seguintes palavras-chave: lavras - chave: satisfação dos usuários; Odontologia; Sistema Único de Saúde (SUS) para acesso às Bases de dados Literatura Latino-Americana e do Caribe em Ciências da Saúde (LILACS), e SCIELO.

O período a ser pesquisado compreende de 1988 a 2018, com intuito de se ter acesso aos trabalhos mais recentes sobre o assunto em questão.

Serão incluídos somente artigos científicos, dentro da temática proposta, selecionado por meio da leitura do título e do resumo, com textos completos, publicados na línguas portuguesa e inglesa. Por sua vez, serão adotados os seguintes critérios de exclusão: trabalhos em outro formato que não artigo, como teses e dissertações; os que fogem da temática proposta, artigos incompletos e publicados em outros idiomas que não os citados anteriormente.

Será realizada uma triagem dos artigos, utilizando uma ficha específica para anotação de dados relevantes dos estudos selecionados, para facilitar a categorização destes e a apresentação da síntese do conhecimento. Os artigos com duplicidade em bases de dados distintas serão analisados apenas uma vez.

Após a seleção, os dados dos artigos incluídos serão anotados, em ficha específica, para posterior análise. Os dados de cada artigo a serem registrados serão: título; primeiro autor; ano de publicação; revista de publicação; ano/período do estudo; país de publicação, idioma; tipo de estudo/desenho do estudo; principais aspectos do estudo.

As referências bibliográficas dos artigos encontrados também serão utilizadas na estratégia de busca deste trabalho.

Após a leitura dos artigos, alguns dados e informações foram sistematizados no quadro 1 . Onde os dados foram analisados de forma descritiva. 
Quadro 1: Principais aspectos dos estudos selecionados

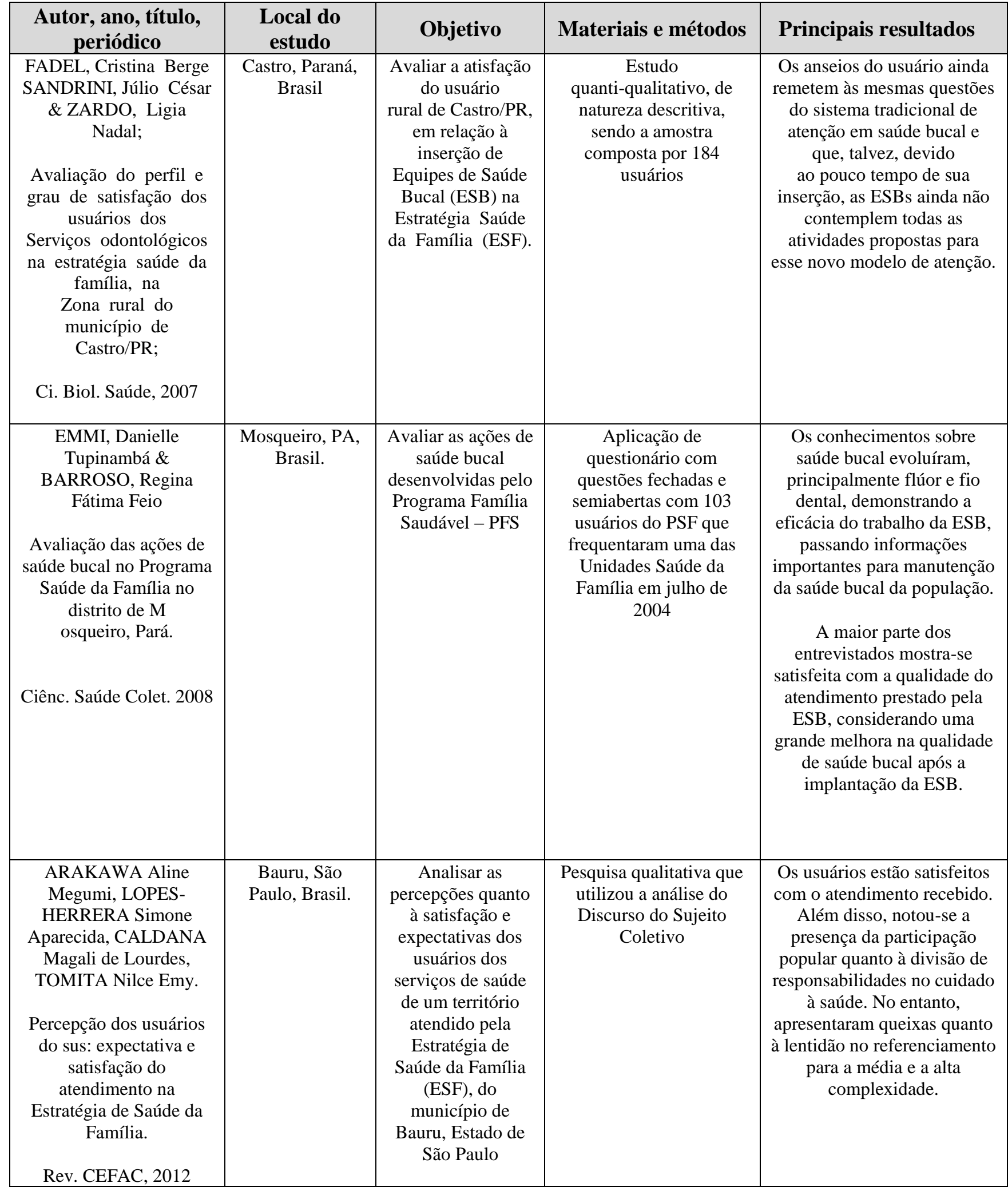




\begin{tabular}{|c|c|c|c|c|}
\hline $\begin{array}{l}\text { RODRIGUES CAQ, et } \\
\text { al; } \\
\text { Factors associated with } \\
\text { satisfaction with dental } \\
\text { services among the } \\
\text { elderly } \\
\text { Rev Saude Publ, } 2012 .\end{array}$ & $\begin{array}{c}\text { Montes Claros, } \\
\text { Brasil }\end{array}$ & $\begin{array}{c}\text { Analisar a } \\
\text { prevalência da } \\
\text { satisfação de } \\
\text { idosos com o } \\
\text { atendimento } \\
\text { odontológico e os } \\
\text { fatores associados. }\end{array}$ & $\begin{array}{c}\text { Estudo transversal com } \\
495 \text { idosos de } 65 \text { a } 74 \\
\text { anos }\end{array}$ & $\begin{array}{l}\text { O idoso que acessa o serviço } \\
\text { ofertado pelo Sistema Único } \\
\text { de Saúde tem maior } \\
\text { satisfação com o tratamento } \\
\text { odontológico, assim como } \\
\text { aqueles que não fazem o uso } \\
\text { de medicamentos, os com } \\
\text { melhor autopercepção da } \\
\text { estética dos dentes e gengivas } \\
\text { e aqueles que possuem mais } \\
\text { acesso a informações sobre } \\
\text { como evitar problemas } \\
\text { bucais. Logo, as políticas de } \\
\text { saúde no SUS apresentam } \\
\text { resultados positivos. }\end{array}$ \\
\hline $\begin{array}{l}\text { BRUNHAUSER, Aline; } \\
\text { MAGRO, Miriam Lago; } \\
\text { NEVES, Matheus; } \\
\text { Avaliação de serviços de } \\
\text { saúde bucal: um estudo } \\
\text { comparativo } \\
\text { Revista da Faculdade de } \\
\text { Odontologia, } 2013\end{array}$ & $\begin{array}{l}\text { Não-me-toque, } \\
\text { RS, Brasil }\end{array}$ & $\begin{array}{l}\text { Avaliar o acesso e } \\
\text { a satisfação dos } \\
\text { usuários dos } \\
\text { serviços } \\
\text { odontológicos do } \\
\text { SUS, comparando } \\
\text { usuários da } \\
\text { Estratégia de } \\
\text { Saúde da Família } \\
\text { (ESF) com } \\
\text { usuários de UBSs } \\
\text { convencionais, em } \\
\text { Não-Me- } \\
\text { Toque/RS, Brasil. }\end{array}$ & $\begin{array}{l}\text { Estudo transversal de } \\
\text { base populacional, com } \\
\text { amostra de } 280 \\
\text { indivíduos }\end{array}$ & $\begin{array}{l}\text { Em Não-Me-Toque/RS, os } \\
\text { usuários cobertos pela ESF } \\
\text { apresentam uma satisfação } \\
\text { maior com os serviços de } \\
\text { saúde bucal que os usuários } \\
\text { cobertos pelas UBSs } \\
\text { convencionais. }\end{array}$ \\
\hline $\begin{array}{c}\text { LAROQUE Mariane } \\
\text { Baltassare, FASSA } \\
\text { Anaclaudia Gastal, } \\
\text { CASTILHOS Eduardo } \\
\text { Dickie de. } \\
\text { Avaliação da atenção } \\
\text { secundária em saúde } \\
\text { bucal do Centro de } \\
\text { Especialidades } \\
\text { Odontológicas de } \\
\text { Pelotas, Rio Grande do } \\
\text { Sul, 2012-2013. } \\
\\
\text { Epidemiol. Serv. Saúde, } \\
\text { 2015 }\end{array}$ & Pelotas, RS & $\begin{array}{l}\text { Avaliar a estrutura, } \\
\text { o processo de } \\
\text { cuidado e os } \\
\text { resultados na } \\
\text { endodontia, } \\
\text { periodontia e } \\
\text { cirurgia realizadas } \\
\text { pelo Centro de } \\
\text { Especialidades } \\
\text { Odontológicas } \\
\text { (CEO) de Pelotas- } \\
\text { RS }\end{array}$ & $\begin{array}{c}\text { Estudo transversal; } \\
\text { avaliou-se o número de } \\
\text { profissionais e equipos } \\
\text { odontológicos do CEO, a } \\
\text { forma de } \\
\text { encaminhamento e } \\
\text { agendamento e o manejo } \\
\text { do absenteísmo; } \\
\text { caracterizou-se a } \\
\text { demanda e os } \\
\text { encaminhamentos para a } \\
\text { central de regulação no } \\
\text { período de julho/2012 a } \\
\text { junho/2013. }\end{array}$ & $\begin{array}{l}\text { O CEO/Pelotas-RS ficou } \\
\text { aquém dos parâmetros de } \\
\text { produção exigidos pelo } \\
\text { Ministério da Saúde e precisa } \\
\text { ampliar sua produção; a } \\
\text { central de regulação necessita } \\
\text { de protocolos que orientem } \\
\text { as prioridades no } \\
\text { atendimento; ademais, são } \\
\text { necessários novos CEO no } \\
\text { município. }\end{array}$ \\
\hline $\begin{array}{c}\text { KITAMURA Elisa } \\
\text { Shizuê, BASTOS } \\
\text { Ronaldo Rocha, PALMA } \\
\text { Pamella Valente, LEITE } \\
\text { Isabel Cristina } \\
\text { Gonçalves. } \\
\\
\text { Avaliação da satisfação } \\
\text { dos usuários dos Centros } \\
\text { de Especialidades } \\
\text { Odontológicas da } \\
\text { macrorregião Sudeste de } \\
\text { Minas Gerais, 2013. }\end{array}$ & $\begin{array}{c}\text { Sudeste de } \\
\text { Minas gerais, } \\
\text { Brasil }\end{array}$ & $\begin{array}{c}\text { Avaliar a } \\
\text { satisfação dos } \\
\text { usuários assistidos } \\
\text { nos Centos de } \\
\text { Especialidades } \\
\text { Odontológicas } \\
\text { (CEO) da } \\
\text { macrorregião } \\
\text { Sudeste de Minas } \\
\text { Gerais, em 2013. }\end{array}$ & $\begin{array}{l}\text { Questionário aplicado } \\
\text { com } 256 \text { pacientes, em } 8 \\
\text { municípios do Sudeste } \\
\text { do Estado de Minas } \\
\text { Gerais }\end{array}$ & $\begin{array}{l}\text { A maioria dos entrevistados } \\
\text { avaliou a atenção recebida } \\
\text { pelo cirurgião-dentista como } \\
\text { excelente, assim como por } \\
\text { outros profissionais do } \\
\text { serviço. Quanto ao } \\
\text { desconforto pós-tratamento, } \\
\text { grande parte relataram não } \\
\text { ter sentido qualquer } \\
\text { incômodo. Na dimensão da } \\
\text { acessibilidade, } 51,6 \% \\
\text { consideraram fácil a obtenção } \\
\text { de vaga para tratamento e }\end{array}$ \\
\hline
\end{tabular}




\begin{tabular}{|c|c|c|c|c|}
\hline $\begin{array}{c}\text { Epidemiol. Serv. Saúde, } \\
2016\end{array}$ & & & & $\begin{array}{l}\text { relataram um tempo de } \\
\text { espera curto }(41,4 \%) \text { no dia } \\
\text { do atendimento }\end{array}$ \\
\hline $\begin{array}{l}\text { MARTINS et al; } \\
\text { Tratamento odontológico } \\
\text { disponível e sua } \\
\text { associação com a } \\
\text { satisfação com os } \\
\text { serviços de atenção } \\
\text { primária à saúde } \\
\text { acessados por idosos } \\
\text { Rev. odontol. } \\
\text { UNESP [Internet], } 2016 . \\
2016 \\
\end{array}$ & $\begin{array}{c}\text { Porto Alegre, } \\
\text { Brasil }\end{array}$ & $\begin{array}{c}\text { Avaliar se a } \\
\text { presença de } \\
\text { equipes de saúde } \\
\text { bucal nas unidades } \\
\text { de atenção } \\
\text { primária à saúde } \\
\text { do SUS está } \\
\text { associada a maior } \\
\text { satisfação com o } \\
\text { serviço acessado. }\end{array}$ & $\begin{array}{c}\text { Entrevistas com } 401 \\
\text { idosos de dois distritos } \\
\text { de Porto Alegre. }\end{array}$ & $\begin{array}{l}\text { As variáveis idade e oferta de } \\
\text { tratamento odontológico se } \\
\text { mantiveram associadas com o } \\
\text { desfecho, já as variáveis } \\
\text { idade, número de dentes e } \\
\text { presença de tratamento } \\
\text { odontológico estiveram } \\
\text { associados com maiores } \\
\text { prevalências à satisfação com } \\
\text { o serviço de saúde. }\end{array}$ \\
\hline $\begin{array}{c}\text { FREITAS CHSM, } \\
\text { LEMOS GA, PESSOA } \\
\text { TRRF, ARAUJO MF, } \\
\text { FORTE FDS. } \\
\text { Atenção em saúde bucal: } \\
\text { avaliação dos centros de } \\
\text { especialidades } \\
\text { odontológicas da } \\
\text { Paraíba. } \\
\text { Saúde em debate, } 2016\end{array}$ & Paraíba, Brasil & $\begin{array}{c}\text { Avaliar a Política } \\
\text { Nacional de Saúde } \\
\text { Bucal na atenção } \\
\text { de média } \\
\text { complexidade, } \\
\text { considerando o } \\
\text { desempenho dos } \\
\text { Centros de } \\
\text { Especialidades } \\
\text { Odontológicas } \\
\text { (CEOs) da Paraíba }\end{array}$ & $\begin{array}{c}\text { Estudo transversal, } \\
\text { quantitativo, de caráter } \\
\text { avaliativo normativo, da } \\
\text { integralidade do cuidado } \\
\text { em saúde bucal na } \\
\text { atenção de média } \\
\text { complexidade da } \\
\text { Paraíba, no período de } \\
\text { 2007 a } 2010 .\end{array}$ & $\begin{array}{l}\text { A análise do cumprimento } \\
\text { das metas, segundo o tipo de } \\
\text { CEO, apontou que, dentre os } \\
\text { CEOs tipo I, nenhum teve } \\
\text { desempenho ótimo nos anos } \\
\text { de } 2007,2008 \text { e } 2009 \text {. No } \\
\text { entanto, no ano de } 2010, \\
\text { apenas um CEO tipo I atingiu } \\
\text { tal desempenho. Nenhum } \\
\text { CEO tipo II apresentou } \\
\text { desempenho ótimo nos } \\
\text { quatro anos analisados. Já o } \\
\text { CEO tipo III exibiu um } \\
\text { desempenho bom no ano de } \\
2007 \text { e ótimo em } 2008,2009 \\
\text { e } 2010 \\
\end{array}$ \\
\hline $\begin{array}{c}\text { BORDIN et al; } \\
\text { Estudo comparativo da } \\
\text { satisfação de usuários e } \\
\text { profissionais da saúde } \\
\text { com o serviço público } \\
\text { odontológico } \\
\text { Ciênc. Saúde Colet;2017 }\end{array}$ & Paraná, Brasil & $\begin{array}{c}\text { Comparar a visão } \\
\text { de usuários com a } \\
\text { de profissionais, } \\
\text { envolvidos direta } \\
\text { ou indiretamente } \\
\text { com a saúde bucal, } \\
\text { acerca da } \\
\text { satisfação com } \\
\text { aspectos inerentes } \\
\text { ao serviço público } \\
\text { odontológico } \\
\end{array}$ & $\begin{array}{c}\text { Pesquisa de caráter } \\
\text { transversal, desenvolvida } \\
\text { na zona urbana de um } \\
\text { município de médio } \\
\text { porte do estado do } \\
\text { Paraná, com população } \\
\text { estimada de } 311.611 \\
\text { habitantes, } \\
\text { predominantemente } \\
\text { urbana e de perfil adulto- } \\
\text { jovem }\end{array}$ & $\begin{array}{l}\text { De modo geral, os usuários } \\
\text { relataram estar satisfeitos } \\
\text { com o serviço público } \\
\text { odontológico ofertado. O } \\
\text { grupo de ACS expôs menor } \\
\text { satisfação do que usuários, } \\
\text { apresentando diferença } \\
\text { significativa para as } \\
\text { variáveis: 'qualidade do } \\
\text { atendimento clínico ofertado } \\
\text { pela equipe de saúde bucal' }\end{array}$ \\
\hline
\end{tabular}

Fonte: Autores 


\section{Resultados}

\section{Perfil dos trabalhos selecionados}

Após acesso às bases de dados foram encontradas 32 publicações no portal LILACS, dos quais seis estavam repetidos e 20 não atendiam aos critérios pré-estabelecidos por não tratarem da temática em questão, portanto foram selecionados seis artigos dessa base. Na busca realizada no SCIELO foram encontradas 48 publicações das quais apenas três atendiam todos os critérios estabelecidos.

No total foram contempladas neste estudo nove publicações (quadro 1). Todos os artigos foram publicados em periódicos, sendo oito em revistas brasileiras diversas e apenas uma em revista estrangeira.

Quanto ao local de realização dos estudos, dos dez, cinco são da região Sul (50\%), 3 da Região Sudeste (30\%), 1 da região Norte (10\%) e 1 da Região Nordeste (10\%).

Quanto ao período de publicação, variou de 2007, a mais antiga a 2017, a mais recente.

\section{Satisfação dos usuários da atenção básica}

Um questionário aplicado aleatoriamente, em quatro distritos rurais no município de Castro no Paraná, constatou a satisfação usuários dos serviços odontológicos em receber o atendimento odontológico, por meio de Equipe de Saúde Bucal (ESB) no Programa de Saúde da Família (PSF). Dos 184 usuários, participantes da pesquisa, 50,5\% estavam satisfeitos com o cirurgião-dentista, $36 \%$ mostraram-se muito satisfeitos, $10,8 \%$ relataram estar mais ou menos satisfeitos e $2,71 \%$, insatisfeitos. Na questão do entendimento das orientações repassadas pelo dentista ou auxiliar, a maioria dos entrevistados (61,4\%) respondeu compreenderam; 33,1\% não compreenderam e 5,43\% entenderam, algumas vezes, os termos usados pelos profissionais (FADEL; SANDRINI e ZARDO, 2007)

Pesquisa realizada em comunidade no Pará, por meio de aplicação de questionário com usuários dos serviços do PSF indicou resultados satisfatórios. Para os entrevistados houve 
evolução dos conhecimentos sobre saúde bucal, especialmente flúor e fio dental, demonstrando a eficácia do trabalho da equipe, com informações importantes para manutenção da saúde oral da população. A maior parte dos entrevistados mostraram-se satisfeitos com a qualidade do atendimento prestado e apontou melhora na qualidade da saúde bucal das pessoas (EMMI et al, 2008).

Em Bauru, no estado de São Paulo, foi analisada a satisfação e expectativas dos usuários atendidos por uma unidade da Estratégia de Saúde da Família, na qual se contava com ESB, através de uma pesquisa qualitativa com 10 usuários. Foi verificado que estes mostram-se satisfeitos com o atendimento recebido, entretanto tinham queixas com relação ao tempo necessário para o atendimento em casos de média e alta complexidade (ARAKAWA Aline Megumi, LOPES-HERRERA Simone Aparecida, CALDANA e TOMITA, 2012).

Estudo transversal realizado com 495 idosos na cidade de Montes Claros, Minas Gerais, analisou a satisfação dos usuários dos serviços odontológicos do SUS. Foram observados resultados satisfatórios. Notou-se relevância das políticas de saúde que promoviam acesso a informação de como evitarem problemas bucais bem como melhores técnicas de escovação, aumentando assim a satisfação destes usuários com os serviços prestados pelo SUS (RODRIGUES et al, 2012).

Outro estudo transversal comparativo realizado com 280 pessoas no município de Nãome-toque, comparou o nível de satisfação de usuários das ESF com o das Unidades Básicas de Saúde (UBS). 93\% dos usuários das Estratégias de Saúde da Família mostraram-se muito satisfeitos com os serviços, enquanto que nos usuários das UBS esse nível de satisfação foi de $86 \%$, demonstrando que em uma perspectiva geral ambos estão satisfeitos com os serviços prestados (BRUNHAUSER; MAGRO e NEVES, 2013).

Pesquisa realizada, em dois distritos de Porto Alegre, avaliou se presença de equipes de Saúde Bucal nas unidades básicas estaria associado a um maior grau de satisfação por parte dos usuários. Os resultados mostraram a necessidade da interação de equipe de saúde bucal às equipes de APS, pois o contentamento do usuário é relativamente proporcional à presença deste grupo nas unidades (MARTINS et al, 2016).

Entrevista realizada com usuários e profissionais diretamente e indiretamente envolvidos com a saúde bucal, em município de médio do porte no Paraná, analisou a satisfação com o serviço público odontológico prestado na região, sob a visão dos usuários e dos 
profissionais. E então foi observado que os profissionais e usuários apresentaram similaridade no grau de satisfação (BORDIN et al, 2017).

\section{Satisfação com a utilização de serviços especializados}

Estudo transversal, realizado no período de julho de 2012 a junho de 2013, no município de Pelotas/RS, com objetivo de analisar os resultados da produção do Centro de Especialidades Odontológicas Jequitibá, avaliou juntamente o número de profissionais e equipes disponíveis para uso, além das formas de encaminhamento e agendamento. Constatou que dos 940 pacientes atendidos apenas $76 \%$ haviam tido o seu tratamento concluído no decorrer de aproximadamente 1 ano, onde o tempo médio de espera para o início do tratamento eram de dois meses, e que ainda haviam 849 usuários na fila de espera do atendimento, classificando dessa forma o CEO aquém dos parâmetros exigidos pelo Ministério da saúde, e pouca satisfação por parte dos pacientes uma vez que a espera em $13 \%$ dos casos levou à desistência do tratamento (LAROQUE; FASSA e CASTILHOS, 2015).

Questionário de avaliação da qualidade dos serviços de saúde bucal aplicados a 256 pacientes, em uma macrorregião Sudeste de Minas gerais, no ano de 2013, avaliou o grau de satisfação dos usuários nos Centros de Especialidades Odontológicas (CEO), onde foi constatado que $86,7 \%$ se declararam satisfeitos com o atendimento recebido e apenas $10,2 \%$ estavam pouco satisfeitos, concluindo assim que a maioria mostrava-se contentados com o serviço prestado (KITAMURA; BASTOS; PALMA e LEITE, 2016).

Estudo transversal quantitativo realizado no período de 2007 a 2010, no estado da Paraíba avaliou a Política Nacional de Saúde Bucal de média complexidade constatou que nenhum dos Centros de Especialidades Odontológicas (CEO) do tipo II apresentou um desempenho considerado como ótimo no período da pesquisa, e que apenas no ano de 2010 foi que um dos CEOs tipo I conseguiu atingir tal patamar. Em contrapartida em um dos Centros foi observado que além das produções de média complexidade alguns profissionais também realizam determinados procedimentos de maior complexidade visando ajudar o paciente, fato que foi considerado como positivo na avaliação da humanização do ambiente e relação do profissional com o paciente (FREITAS; LEMOS; PESSOA; ARAUJO e FORTE, 2016).

712 Id on Line Rev. Mult. Psic. V.12, N. 42, p. 704-717, 2018 - ISSN 1981-1179 Edição eletrônica em http://idonline.emnuvens.com.br/id 


\section{Discussão}

Como observado nos estudos de Freitas et. al. (2016) e Bordin et al, (2017), a grande maioria dos participantes dos estudos eram do sexo feminino, o que confirma a pesquisa de Corbacho (2011), onde 78,2\% dos entrevistados eram mulheres e foi atribuído este resultado ao fato do sexo feminino frequentar mais as unidades de Estratégia de Saúde da Família (ESF) para exames de rotina e prevenção, enquanto o sexo masculino busca por atendimento apenas em casos extremos.

No que se refere à avaliação das relações humanas nas unidades de atendimento do SUS, em nos estudos ${ }^{13-21}$ constatou-se um elevado nível de satisfação dos usuários, onde destacou-se os estudos de Kitamura et al. (2016), onde 87,6\% dos pacientes entrevistados classificaram o atendimento como excelente, e o de Medeiros e colaboradores (2010) onde os usuários consideraram pontos relevantes e positivos a resolutividade no atendimento, a humanização dos funcionários e a solidariedade destes.

No quesito da disponibilização dos serviços e o tempo necessário para a marcação de uma consulta, no estudo de Kitamura et. al. (2016), pode-se observar que a maioria dos entrevistados consideraram que há falhas, considerado como difícil ou muito difícil por quase $70 \%$ da população usuária .

Confirmando os achados de outros estudos ((D’ÁVILA et. al., 2010; FADEL; SANDRINI e ZARDO, 2007; ARAKAWA et. al., 2012), a maioria dos pacientes consideram a estrutura física das unidades de saúde como satisfatórias, bem higienizadas e organizadas (ARAKAWA, et al., 2012).

Ao considerar o baixo nível socioeconômico, da maioria, dos usuários do atendimento odontológico do SUS, o alto nível de satisfação pode ser questionado, uma vez que estes não dispõem de outros tipos de serviços odontológicos que possam servir como parâmetro de comparação, supervalorizando assim o atendimento recebido (CORBACHO, et al., 2011; D’AVILA et al., 2010).

Estudo realizado por Bordin e colaboradores (2017), ressalta que resultados devem ser analisados com cautela, pois os usuários de saúde tendem a expor certo conformismo em relação aos serviços, baixo teor crítico e reivindicativo e ainda uma visão equivocada no reconhecimento do serviço de saúde como um benefício e não como um direito. Isso pode levar 
à falsa alta qualidade dos serviços de saúde pesquisados ${ }^{7}$. É importante a construção de espaço no qual os usuários possam compreender e exercitar os seus direitos, bem como desenvolver uma visão crítica em relação às suas necessidades e do processo de trabalho nas unidades de saúde.

Brunhauser, Magro e Neves (2013), ao realizarem seus estudos perceberam a influência da interação da equipe de saúde com o nível de satisfação e adesão ao tratamento ou às ações preventivas. As relações interpessoais no cuidado, melhoram o desfecho terapêutico. Torna-se importante incorporar a avaliação da satisfação do usuário como um dos componentes da qualidade do serviço. Apesar da literatura disponível afirmar que a ESF proporciona aumento da satisfação dos usuários é necessário refletir se de fato as mudanças no modelo assistencial em saúde bucal, caracterizado pelo atendimento individualizado, na consulta e no procedimento que visa apenas ao tratamento e resiste à incorporação do conceito de cuidado em saúde. Acrescentei essa parte desse artigo para a gente utilizar na discussão.

Notou-se relevância das políticas de saúde que promoviam acesso a informação de como evitarem problemas bucais bem como melhores técnicas de escovação, aumentando assim a satisfação destes usuários com os serviços prestados pelo SUS pode ser para a discussão a parte em laranja (RODRIGUES et al, 2012).

No estudo de Arakawa et al (2012), destacam a elevada satisfação dos usuários atendidos no PSF, mas é importante pontuar a dificuldade das equipes em realizar o encaminhamento das pessoas para a atenção secundária, a exemplo de endodontia e prótese. A oferta de serviços pelos Centros de Especialidades Odontológicas e outros serviços do SUS de média complexidade fica muito aquém das necessidades das pessoas.

Os resultados apresentados por Emmi e Barroso (2018) evidenciam a importância da integralidade na atenção em saúde, tendo-se em vista que o trabalho realizado no PSF não se restringe à atividades curativas. As maiores satisfações dos usuários foram relacionadas às orientações de higiene oral, melhor acesso ao atendimento e visitas domiciliares

Como observado por Fadel, Sandrini e Zardo (2007), acolhimento do usuário e a construção de vínculos melhora as relações entre os profissionais e a comunidade e amplia a aceitação do trabalho realizado pela equipe. Tudo isso reflete positivamente para a satisfação dos usuários. 


\section{Conclusões}

Levando em consideração a metodologia empregada e as limitações deste estudo, foi possível concluir que:

- Houve um bom grau de satisfação por parte dos usuários de todos os tipos de serviços dos SUS, tanto os especializados quanto o de atenção básica;

- Os quesitos de área física, incentivo à participação popular, demanda reprimida e tempo de espera no referenciamento em casos de média e alta complexidade foram citados como pontos negativos;

- Apesar dos desafios para a melhoria da qualidade no atendimento e atenção à saúde bucal nas UBSs e ESFs, a satisfação dos usuários pode ser citada como excelente, uma vez que todos se declararam contentados com pelos menos três quesitos de avaliação;

Sugere-se a realização de outros estudos a fim de aprofundar a compreensão da realidade dos serviços odontológicos prestados em cada região, comparando-as, visando dessa formar fornecer melhores subsídios para o desenvolvimento de Políticas Nacionais de Saúde que supram cada vez mais as necessidades populacionais, levando em conta as expectativas e percepções dos usuários desses sistemas de saúde.

\section{Referências}

ARAKAWA Aline Megumi, LOPES-HERRERA Simone Aparecida, CALDANA Magali de Lourdes, TOMITA Nilce Emy. Percepção dos usuários do sus: expectativa e satisfação do atendimento na Estratégia de Saúde da Família. Rev. CEFAC [Internet]. 2012; 14( 6 ): 11081114

Atlas Brasil - Programa das Nações Unidas para o Desenvolvimento. Caracterização do território do Município de Paramirim-Ba 2013. [Acesso em: 2017 Abr 12]. Disponível em: < http://atlasbrasil.org.br/2013/pt/perfil_m/paramirim_ba>.

BORDIN D, FADEL CB, SALIBA AS, MOIMAZ SAS, GARBIN CAS, SALBIA NA. Estudo comparativo da satisfação de usuários e profissionais da saúde com o serviço público odontológico. Cienc \& Saud Coletiva. 2017; 22(1): 151-60

BORDIN, Danielle et al. Estudo comparativo da satisfação de usuários e profissionais da saúde com o serviço público odontológico. Ciênc. Saúde Colet, vol.22, n.1, pp.151-160. 2017. 
BRASIL. Ministério da Saúde. Instrumento de Avaliação Externa para as Equipes da Atenção Básica. Brasília: Ministério da Saúde, 2013.

BRUNHAUSER, Aline; MAGRO, Miriam Lago; NEVES, Matheus. Avaliação de serviços de saúde bucal: um estudo comparativo. Revista da Faculdade de Odontologia, Passo Fundo, v. 18, n. 1, abr. 2013.

CNES - Cadastro Nacional de Estabelecimento de Saúde. Secretaria de Atenção à Saúde. [Acesso em: 2017 Abr 10]. Disponível em: <CNES

http://cnes2.datasus.gov.br/Lista_Es_Nome.asp?Vbusca=paramirim\&VTipo=1>.

CORBACHO, MM. et al. Percepção de Saúde Bucal: Uma análise de famílias participantes do programa de Saúde Salvador-Ba, Revista da Faculdade de Odontologia da UFBA, Salvador, v.22, jan/jun 2011, p.6-11.

D’AVILA „S.; SOUZA, M M .; CAVALCANTI,GMS. LUCAS, R S C C.; GRANVILLEGARCIA, A F.; CAVALCANTI, A L. Nível de satisfação e condição socioeconômica dos usuários das clínicas de odontologia da Universidade Estadual da Paraíba. Revista Brasileira de Pesquisa em Saúde 2010; 12(2) : 39-45

DONABEDIAN, A. The sevenpillarsofquality. Arch. Pathol. Lab. Med., Michigan. 1990; 114: 1115-8.

DONABEDIAN,A.; WHEELER,J.R.C.; WYSZEWIANSKI,L. Quality, Cost, and Health: An Integrative Model. Medical Care. v. XX, n. 10, October, 1982

EMMI Danielle Tupinambá, BARROSO Regina Fátima Feio. Avaliação das ações de saúde bucal no Programa Saúde da Família no distrito de Mosqueiro, Pará. Ciênc. saúde coletiva [Internet]. 2008; 13( 1 ): 35-41

FADEL, Cristina Berge SANDRINI, Júlio César \& ZARDO, Ligia Nadal. Avaliação do perfil e grau de satisfação dos usuários dos serviços odontológicos na estratégia saúde da família, na zona rural do município de Castro/PR. Ci. Biol. Saúde. 2007; 13(3/4): 59-66.

FREITAS CHSM, LEMOS GA, PESSOA TRRF, ARAUJO MF, FORTE FDS. Atenção em saúde bucal: avaliação dos centros de especialidades odontológicas da Paraíba. Saúde debate [Internet]. 2016; 40(108): 131-43

IBGE - Instituto Brasileiro de Geografia e Estatística. Pesquisa sobre estimativa da população 2016. [Acesso em: 2017 Abr 12]. Disponível em: <http://cidades.ibge.gov.br/xtras/perfil.php?codmun=292360>.

KITAMURA Elisa Shizuê, BASTOS Ronaldo Rocha, PALMA Pamella Valente, LEITE Isabel Cristina Gonçalves. Avaliação da satisfação dos usuários dos Centros de Especialidades

716 Id on Line Rev. Mult. Psic. V.12, N. 42, p. 704-717, 2018 - ISSN 1981-1179 Edição eletrônica em http://idonline.emnuvens.com.br/id 
Odontológicas da macrorregião Sudeste de Minas Gerais, 2013. Epidemiol. Serv. Saúde [Internet]. 2016; 25( 1 ): 137-148

LAROQUE Mariane Baltassare, FASSA Anaclaudia Gastal, CASTILHOS Eduardo Dickie de. Avaliação da atenção secundária em saúde bucal do Centro de Especialidades Odontológicas de Pelotas, Rio Grande do Sul, 2012-2013. Epidemiol. Serv. Saúde [Internet]. 2015; 24( 3 ): 421-430.

MALLET ALR. Health Care Quality: topics for discussion. Rev. Soc. de Cardiologia. 2(1); 203: 2011.

MARTINS Aline Blaya, SEIBT Creta, NEVES Matheus, HILGERT Juliana Balbinot, HUGO Fernando Neves. Availability of dental treatment is associated with satisfaction derived from Primary Health Care Services accessed by elderly. Rev. odontol. UNESP [Internet]. 2016 Dec [cited 2018 Oct 04] ; 45( 6 ): 344-350

MATOS PES, TOMITA NE. A Inserção da saúde bucal no Programa Saúde da Família: da universidade aos pólos de capacitação. Cad. Saúde Pública v.20 n.6 Rio de Janeiro Nov./dez.2010.

MOYSÉS, S.J. O conceito de Promoção da Saúde na Construção de Sistemas de Atenção em Saúde Bucal Coletiva. In: KRIGER, L. ABOPREV. Promoção de Saúde Bucal. Artes Médicas, 2a ed., cap 16, p.373-471, 2009.

OMS. Conferência Internacional sobre Cuidados Primários de Saúde: Declaração de AlmaAta, 1978. Brasília, DF: Ministério da Saúde, 2004

RODRIGUES CAQ, et al. Factors associated with satisfaction with dental services among the elderly. Rev Saud Publ. 2012; 46(6): 1039-50

\section{Como citar este artigo (Formato ABNT):}

AZEVEDO, Luciana Cristina Salvador; ROCHA, Marcelo Pereira da. Satisfação dos usuários de serviços públicos odontológicos: Revisão da literatura. Id on Line Rev.Mult. Psic., 2018, vol.12, n.42, p. 704-717. ISSN: 1981-1179.

Recebido: 11/10/2018;

Aceito: 16/10/2018 